\title{
Phenolic Acid and Flavonoid Patterns in Twelve Sechium edule Varieties
}

\section{Jyothi Ramesh Jain ${ }^{1 *}$, Shiragambi Hanumantgowda Manohar ${ }^{1}$, Tapas Kumar Roy ${ }^{2}$ and Kumudini Belur Satyan ${ }^{1}$}

${ }^{1}$ Department of Biotechnology, JAIN (Deemed_To_Be_University), School of Sciences, Bangalore, India

${ }^{2}$ Division of Plant Physiology and Biochemistry, ICAR-Indian Institute of

Horticultural Research, Bangalore, India

*Corresponding Author: Jyothi Ramesh Jain, Department of Biotechnology, JAIN

(Deemed_To_Be_University), School of Sciences, Bangalore, India
Received: January 29, 2021

Published: March 09, 2021

(C) All rights are reserved by Jyothi Ramesh

Jain., et al.

\begin{abstract}
Fruit pulp of twelve Sechium edule Indian accessions were analyzed for phenolic acid and flavonoid constituents. The quantitative evaluation was performed using liquid chromatography mass spectrophotometer method, which showed significant differences in the composition of phenolic acids and flavonoids among accessions. Vanillic acid was the predominant phenolic acid in most of the accessions ranging from 269.28 to $4080.82 \mu \mathrm{g} / \mathrm{g}$. High amounts of vanillic acid in accession SEC-11 (4080.82 $\pm 130.92 \mu \mathrm{g} / \mathrm{g})$ and SEC-06 (1825.46 $\pm 24.54 \mu \mathrm{g} / \mathrm{g})$, protocatechuic acid (1736.59 $\pm 94.90 \mu \mathrm{g} / \mathrm{g})$ in SEC-09 and syringic acid (1676.97 $\pm 70.35 \mu \mathrm{g} / \mathrm{g})$ in SEC-20 was detected respectively. The highest amount of flavonoid present was catechin in the accessions SEC-36 (75.83 $\pm 4.37 \mu \mathrm{g} / \mathrm{g}$ ) followed by SEC-20 (19.43 $\pm 0.64 \mu \mathrm{g} / \mathrm{g})$. Data were analyzed using principal component analysis method and the obtained scoring plot showed that all nine accessions had formed one cluster. Discrimination of metabolic profiles of different $S$. edule accessions using principal component analysis showed that accessions grouping was consistent with the LC-MS results obtained. This method of estimation of metabolites can be successfully employed enabling genetic grouping of $S$. edule accessions in an effective manner for breeding studies.
\end{abstract}

Keywords: Sechium edule; Phenolic Acids; Flavonoid; Principal Component Analysis; LCMS

\section{Introduction}

The health promoting benefits in Sechium edule fruits can be attributed to the presence of phytochemicals and large number of plant food derived bioactive compounds belongs to phenolic acid and flavonoid families [1]. Therefore, phenolic acids and flavonoids are two such secondary metabolites, which needs to be investigated. Phenolic acids, which are produced by shikimic acid pathway, are present as free form or are conjugated with sugar residue. They are classified as hydroxycinnamic acids and hydroxybenzoic acids based on the carbon framework. These also arise in plants in form of glycosides or esters with other compounds like sterols, glucosides or alcohols [2]. The cinnamic acid deriva- tives are sinapic acid, coumaric acid, ferulic acid and caffeic acid and the hydroxybenzoic acids are protocatechuic acid, gallic acid, vanillic acid and syringic acid with a C6-C1 configuration. Phenolic acids are of utmost importance recently due to their protective role against cancer and heart diseases, which may be attributed to their antioxidant activity, reported to be higher than vitamin $\mathrm{C}$ and E against reactive oxygen species [3].

On the other hand, flavonoids belong to a family of C6-C3-C6 polyphenol compounds and the subclasses includes flavone, flavonol, flavanone, flavanol, anthocyanin and isoflavone. More than 8000 flavonoids have been identified so far [4] and the number 
continues to grow. They play a very important role in protecting plants against insect and microbial attack and possess remarkable health promoting effects such as, anti-oxidant [5], anti-microbial [6], anti-cancer [7] activity. They also help in the prevention of osteoporosis [8]. In view of the impact of both phenolic acids and flavonoids on human health, it is pivotal to learn about their concentrations and variations in medicinal plants.

Sechium edule, a lesser-known vegetable crop from the cucurbit family, is cultivated from the pre- Columbian times. The fruits, roots as well as stem has been important elements of the diet of the people throughout the world. The fruits grow best in tropical regions across the world and are indigenous to Mexico and Central America [9]. S. edule displays a wide diversity and produces fruits with different colors, shapes and sizes depending on the cultivar. There has been a decline in the number of accessions in the past. Conservation of such species by orthodox methods has not been possible so far as the seeds of the fruit are recalcitrant and viviparous in nature [10]. Sechium genetic resources have been assessed for characterization of the germplasm but characterization of phenolic acids and flavonoids among different accessions has been limited to studies of role of phenolic acids against melon fly and flavonoids detection in leaves, stem and fruits [11,12]. The studies have shown the presence of different pharmacological components like peroxidases, phenols, alkaloids, flavonoids, saponins and tannins which are potent anticancer compounds (Firdous., et al. 2012; Lombardo-Earl., et al. 2014). No studies have been carried out on S. edule regarding the potential that these might have as a resource for improving this species. As it is diverse in nature, these populations should be evaluated and for hybridization programmes with cultivated types needs to be stated.

In the present study, liquid chromatography coupled with mass spectrometry (LCMS) was used for the quantitative determination of phenolic acids and flavonoids of $S$. edule fruit extracts of 12 diverse accessions. Since very few species have been explored for its phytochemicals across India, this work will draw attention towards this species and contribute for the potential value and improvement of this underutilized and neglected crop for developing varieties with desired nutrients.

\section{Materials and Methods}

Chemicals and reagents

The phenolic acids and flavonoids standards used in the present study were purchased from Sigma Aldrich, USA and these stan- dards were prepared with a range of $1 \mathrm{mg} / \mathrm{ml}$ using $80 \%$ methanol. Chromatographic grade organic solvents were used for the analysis and Milli-Q (Millipore) purification system was used to obtain purified water for preparing mobile phases and the extracts were filtered through $0.45 \mu \mathrm{m}$ membrane filters.

\section{Sample preparation and extraction}

The place of collection of all the accessions of $S$. edule collected across India have been represented (Table 1) and the phenotypic variations is depicted in the Figure 1. Phenolic acids and flavonoids were extracted from $5 \mathrm{~g}$ of $S$. edule as per protocol described by Weidner., et al. [13], Chen., et al. [14] and Middha., et al. [15] with slight modification.

\begin{tabular}{|l|c|c|}
\hline \multicolumn{1}{|c|}{$\begin{array}{c}\text { Accession } \\
\text { Number }\end{array}$} & $\begin{array}{c}\text { Place of collection } \\
\text { (State) }\end{array}$ & District \\
\hline SEC-01 & Sikkim & Lingzey \\
\hline SEC-03 & Sikkim & Gangtok \\
\hline SEC-05 & Sikkim & Ganktok \\
\hline SEC-06 & Assam & Kamrup \\
\hline SEC-09 & Meghalaya & Shillong \\
\hline SEC-11 & Manipur & Senapati \\
\hline SEC-13 & Manipur & Ukhrul \\
\hline SEC-18 & Mizoram & Aizawl \\
\hline SEC-20 & Karnataka & Bangalore \\
\hline SEC-27 & Tamil Nadu & Ooty \\
\hline SEC-31 & Kerala & Idukki \\
\hline SEC-36 & &
\end{tabular}

Table 1: S. edule accessions used with code and collection site.

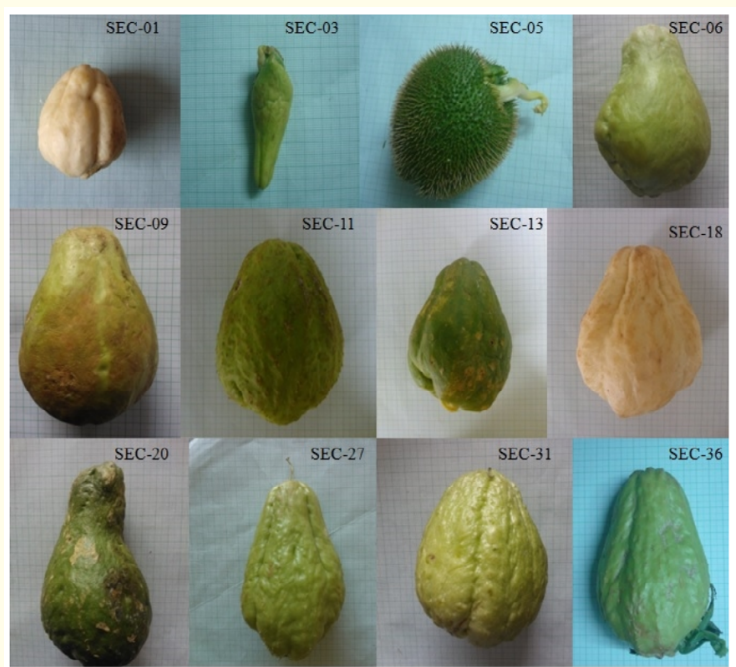

Figure 1: Picture showing the diversity of fruit size, shape and color for Sechium edule accessions collected from India. 
The fruit pulp was homogenized two times using $80 \%$ methanol and was centrifuged, the supernatant was collected and made up to $80 \mathrm{ml}$ with $80 \%$ methanol. The $20 \mathrm{ml}$ of $80 \%$ methanol extract was evaporated in a rotary evaporator at $50^{\circ} \mathrm{C}$ till the methanol completely evaporates. It is then extracted three times with ethyl acetate. The ethyl acetate layer was then evaporated to dryness at room temperature under vacuum. To the water residue, $2 \mathrm{~N} \mathrm{NaOH}$ ( $4 \mathrm{ml}$ ) was added and allowed to be hydrolyzed overnight at room temperature. After acidification to $\mathrm{pH} 2$ using $2 \mathrm{~N} \mathrm{HCl}$, the extracts were once again subjected to ethyl acetate extraction. Discarding the aqueous layer, the ethyl acetate layer was further extracted two times with $20 \mathrm{ml}$ of $0.1 \mathrm{~N} \mathrm{NaHCO}_{3}$.

The aqueous layer was further acidified to pH 2 with $5 \mathrm{ml} 2 \mathrm{~N}$ $\mathrm{HCl}$ and extracted three times with $25 \mathrm{ml}$ ethyl acetate. The ethyl acetate layer was then washed with distilled water till the $\mathrm{pH}$ becomes 6.5 to 7. It was then dried completely in rotary evaporator and the residue was dissolved in $1 \mathrm{ml}$ MS grade methanol, filtered through $0.2 \mu \mathrm{m}$ nylon membrane filter prior to inject in LCMS for quantification of phenolic acids.

The ethyl acetate layer, which carried the flavonoids was washed with water several times till the $\mathrm{pH}$ becomes 6.5 to 7 . It was then evaporated to complete dryness under vacuum at room temperature. The residue was dissolved in $1 \mathrm{ml}$ MS grade methanol filtered through $0.2 \mu \mathrm{m}$ nylon membrane filter before injecting into LCMS for quantification of flavonoids.

\section{Equipments}

LCMS analysis were carried out using a Waters Acquity UPLC-H class coupled with TQD-MS/MS (Waters Corp, USA), which was equipped with a quaternary pump, degasser and a diode array detector along with temperature control system for analytical column. This was accompanied with an electro spray ionization (ESI) source for phenolic acids and flavonoids quantification. The automatic injection system having a range of $(0-10 \mu \mathrm{L})$ and the overall system was controlled by Mass lynx software for data collection. The electro spray ionization source was operated in negative ion mode (ES-) to detect parent mass $\mathrm{m} / \mathrm{z}$ and most abundant fragmented daughters of phenolic acids and flavonoids by MRM method in LC-MS. Calibration curve were obtained by using different concentrations for individual phenolic acids and flavonoids.
Liquid chromatography-Mass spectrometry (LC-MS) conditions

A liquid chromatography separation was performed using analytical column 2.1 X 50 mm UPLC BEH- C18 column (Waters) with $1.7 \mu \mathrm{m}$ particles, safeguarded by a Vanguard BEH C-18 with $1.7 \mu \mathrm{m}$ guard column (Waters). The column was set to $25^{\circ} \mathrm{C}$. Aqueous phase of formic acid (0.1\%) + water (A) and organic phase of formic acid $(0.2 \%)+$ methanol (B) was used as mobile phase. The initial gradient had both the phases in the ratio of 90:10 (A:B) and held for 2.5 minutes. The gradient was then changed to $70: 30$ at $4.0 \mathrm{~min}$, then the gradient was reduced to 60:40 after $1 \mathrm{~min}$ and held for $5.0 \mathrm{~min}$ and later brought to $80: 20$ (A:B) held for $2.0 \mathrm{~min}$. It is then finally returned to the initial gradient $90: 10$ in $2.0 \mathrm{~min}$ held for a $1 \mathrm{~min}$, so as to equilibrate, prior to the next injection. The mobile phase flow rate was maintained at $0.3 \mathrm{~mL} / \mathrm{min}$ and 4 $\mu \mathrm{l}$ of sample was injected each time for both phenolic acids and flavonoids.

\section{Statistical analysis}

The assessment of data obtained was performed using principal component analysis (PCA) by using a multivariate software (Unscrambler X, CAMO, Bangalore, Karnataka, India). All the samples were injected thrice into the LCMS system and the data for the sample content are expressed as mean \pm standard deviation. Data collected were analyzed using two-way ANOVA test (GraphPad prism 6.01 ) representing the significant difference at $P<0.05$.

\section{Results and Discussion}

Phenolic acid composition in $S$, edule accessions

Among the phenolic acids we detected fourteen phenolic acids by LC-MS analysis of fruit extracts from twelve accessions of S. edule. The details of multiple-reaction monitoring of phenolic acid standards is given in the Table 2. Chlorogenic acid per dry weight ranged from 0.06 to $0.17 \mu \mathrm{g} / \mathrm{g}$; ferulic acid ranged from 5.44 to $2543 \mu \mathrm{g} / \mathrm{g}$; caffeic acid ranged from 0.43 to $27.61 \mu \mathrm{g} / \mathrm{g}$; gallic acid ranged from 0.46 to $96 \mu \mathrm{g} / \mathrm{g}$; vanillic acid ranged from 269.28 to $4080.82 \mu \mathrm{g} / \mathrm{g}$; $p$-coumaric acid ranged from 50.85 to 1220.81 $\mu \mathrm{g} / \mathrm{g}$; o-coumaric acid ranged from 25.90 to $940.88 \mu \mathrm{g} / \mathrm{g}$; protocatechuic acid ranged from 0.94 to $1736.59 \mu \mathrm{g} / \mathrm{g}$; gentisic acid ranged from 0.12 to $0.67 \mu \mathrm{g} / \mathrm{g}$; salicylic acid ranged 0.17 to $1483.22 \mu \mathrm{g} / \mathrm{g}$; p-hydroxybenzoic acid ranged from 225.07 to $1008.53 \mu \mathrm{g} / \mathrm{g} ; 2,4$ dihydroxybenzoic acid ranged from 0.91 to $5.42 \mu \mathrm{g} / \mathrm{g}$; t-cinnamic acid ranged from 0.49 to $17.36 \mu \mathrm{g} / \mathrm{g}$ and syringic acid ranged from 388.78 to $1676.97 \mu \mathrm{g} / \mathrm{g}$ (Table 3). 


\begin{tabular}{|c|c|c|c|c|c|c|}
\hline Compound & Formula/Mass & Parent m/z & Cone Voltage & Daughters & Collision Energy & Ion Mode \\
\hline Caffeic acid & 180 & 178.90 & 30 & 135.05 & 16 & ES- \\
\hline $\begin{array}{l}2,4 \text { dihydroxybenzoic } \\
\text { acid }\end{array}$ & 154 & 152.90 & 28 & 65.02 & 18 & ES- \\
\hline Chlorogenic acid & 354 & 352.97 & 22 & 191.10 & 18 & ES- \\
\hline Ferulic acid & 194 & 192.90 & 26 & 134.02 & 14 & ES- \\
\hline Gallic acid & 170 & 168.90 & 28 & 125.03 & 12 & ES- \\
\hline Gentisic acid & 154 & 152.90 & 24 & 108.98 & 12 & ES- \\
\hline$o$-Coumaric acid & 164 & 162.90 & 22 & 119.06 & 12 & ES- \\
\hline$p$-coumaric acid & 164 & 162.90 & 24 & 119.05 & 14 & ES- \\
\hline $\begin{array}{l}p \text { - hydroxybenzoic } \\
\text { acid }\end{array}$ & 138 & 136.90 & 26 & 93.01 & 12 & ES- \\
\hline Protocatechuic acid & 154 & 152.90 & 26 & 109.05 & 16 & ES- \\
\hline Salicylic acid & 138 & 136.90 & 28 & 93.10 & 14 & ES- \\
\hline Syringic acid & 198 & 196.97 & 26 & 182.07 & 10 & ES- \\
\hline$t$-cinnamic acid & 148 & 146.90 & 26 & 103.05 & 10 & ES- \\
\hline Vanillic acid & 168 & 166.97 & 26 & 108.01 & 20 & ES- \\
\hline
\end{tabular}

Table 2: Phenolic acids MRM details.

Majorly all twelve accessions were rich in vanillic acid, syringic acid and $p$-hydroxybenzoic acid respectively. Syringic acid and hydroxybenzoic acid are abundantly present in fruits and vegetables. They are known for anti-cancer, sedative, anti-proliferative and decongestant properties [16]. Another most important phenolic acid is ferulic acid and $p$-coumaric acid, which is well known for its anti-microbial, anti-inflammatory, anti-cancer activities, lowers cholesterol, and enhances sperm viability $[17,18]$.

Vanillic acid was predominant in accession SEC-11 (4080.82 \pm $130.92 \mu \mathrm{g} / \mathrm{g}$ ) and SEC-18 (2339.45 \pm 16.36$)$, ferulic acid and protocatechuic acid were rich in accession SEC-09 (2543.73 \pm 68.17 $\mu \mathrm{g} / \mathrm{g}$ and $1736.59 \pm 94.90$ ) respectively. The chromatogram of vanillic acid for the extract of $S$. edule accession SEC-11 is represented with the standard curve obtained using multiple reaction monitoring is provided in Figure 2. The total of highest phenolic acids was found in SEC-09 followed by SEC-11 and SEC-20. Lowest amount of phenolic acids found among accessions were chlorogenic and gentisic acid. In a study conducted by Shivashankar et

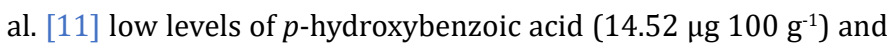
salicylic acid was detected using HPLC detection method $(177.5 \mu \mathrm{g}$ $100 \mathrm{~g}^{-1} \mathrm{FW}$ ) in healthy tissue of $S$. edule fruits. Our findings on the content of phenolic acid of $S$. edule provides the scope for improvement of the quality of fruit and its nutritive value. The insight of gene regulation will help promote or aid the breeding of new cultivars/varieties with any specific phenolic acid composition. Similar studies were conducted in Eggplant from accessions in the USDA eggplant core subset [19]. Comparatively, we could detect higher amounts of phenolic acids in methanolic extracts of $S$. edule. The distribution profile of each phenolic acid among all accessions decreased in the order SEC-09 $>$ SEC-11 $>$ SEC-20 $>$ SEC-18 $>$ SEC-06 $>$ SEC-13 > SEC-03 > SEC-31 > SEC-01 > SEC-05 > SEC-36 > SEC-27.

\section{Flavonoid composition in S, edule accessions}

With the standards available, we could detect nine flavonoids for the methanolic extracts of twelve S. edule accessions. The details of multiple-reaction monitoring of flavonoid standards is given in the Table 4. Quercetin per dry weight ranged from 1.25 to 11.70 $\mu \mathrm{g} / \mathrm{g}$; hespertin ranged from 0.75 to $8.72 \mu \mathrm{g} / \mathrm{g}$; catechin ranged from 2.53 to $75.83 \mu \mathrm{g} / \mathrm{g}$; luteolene ranged from 0.27 to $4.49 \mu \mathrm{g} / \mathrm{g}$; naringenin ranged from 0.18 to $1.36 \mu \mathrm{g} / \mathrm{g}$; apigenin ranged from 0.13 to $0.88 \mu \mathrm{g} / \mathrm{g}$; umbeliferon 0.14 to $1.02 \mu \mathrm{g} / \mathrm{g}$; rutin ranged from 0.28 to $19.43 \mu \mathrm{g} / \mathrm{g}$ and myricetin ranged from 0.48 to $2.08 \mu \mathrm{g} / \mathrm{g}$. 


\begin{tabular}{|c|c|c|c|c|c|c|c|c|c|c|c|c|c|c|}
\hline Acc No. & Chl A & Fer A & Caf A & Gal A & $\operatorname{Van} \mathrm{A}$ & $p$-CA & $o$-CA & Prot A & Gen A & Sal A & $p$-HBA & $\begin{array}{c}2,4 \\
\text { DHBA }\end{array}$ & $t-\operatorname{Cin} \mathbf{A}$ & Syr A \\
\hline C- & $\begin{array}{c}0.17 \pm \\
0.07^{\mathrm{a}}\end{array}$ & $\begin{array}{c}333.99 \pm \\
5.34^{\mathrm{a}}\end{array}$ & $\begin{array}{c}2.61 \pm \\
0.25^{a}\end{array}$ & $\begin{array}{c}96.97 \pm \\
1.60^{\mathrm{a}}\end{array}$ & $\begin{array}{c}659.51 \pm \\
37.64^{\mathrm{a}} \\
\end{array}$ & $\begin{array}{c}127.55 \pm \\
3.39^{\text {al }}\end{array}$ & $\begin{array}{r}97.57 \pm \\
4.04^{\mathrm{ag}}\end{array}$ & $\begin{array}{c}28.34 \pm \\
0.60^{\mathrm{a}}\end{array}$ & $\begin{array}{c}0.12 \pm \\
0.04^{\mathrm{a}}\end{array}$ & $\begin{array}{c}4.99 \pm \\
0.10^{\mathrm{a}} \\
\end{array}$ & $\begin{array}{l}07 \\
2^{\mathrm{a}} \\
\end{array}$ & $0.13^{\mathrm{a}}$ & $\begin{array}{c}12.23 \pm \\
0.57^{\mathrm{a}}\end{array}$ & $\begin{array}{l}130 \\
\pm 20\end{array}$ \\
\hline EC-03 & $\begin{array}{c}0.15 \pm \\
0.03^{\mathrm{a}}\end{array}$ & $\begin{array}{l}1280.56 \\
\pm 44.06^{\mathrm{b}}\end{array}$ & $\begin{array}{c}5.00 \pm \\
0.42^{\mathrm{a}}\end{array}$ & $\begin{array}{c}7.73 \pm \\
0.53^{\mathrm{b}}\end{array}$ & $\begin{array}{l}19.63 \\
2.19^{\mathrm{b}}\end{array}$ & $\begin{array}{c}127.55 \pm \\
3.39^{\mathrm{bij}}\end{array}$ & $\begin{array}{l}145.38 \\
\pm 2.22^{\mathrm{a}}\end{array}$ & $\begin{array}{c}104.46 \pm \\
1.55^{\mathrm{b}}\end{array}$ & $\begin{array}{c}0.27 \pm \\
0.07^{\text {a }}\end{array}$ & $\begin{array}{c}9.81 \pm \\
0.12^{\mathrm{a}}\end{array}$ & $\begin{array}{l}76 \\
70^{\mathrm{b}} \\
\end{array}$ & $\begin{array}{c}0.12 \pm \\
0.05^{\mathrm{a}}\end{array}$ & $\begin{array}{c}16.20 \pm \\
1.43^{\mathrm{a}}\end{array}$ & $\begin{array}{l}8 \pm \\
6^{b}\end{array}$ \\
\hline $\mathrm{E}-\mathrm{O}$ & $\begin{array}{c}0.06 \pm \\
0.03^{\mathrm{a}}\end{array}$ & $\begin{array}{r}5.44 \pm \\
0.63^{\mathrm{c}}\end{array}$ & $\begin{array}{c}0.43 \pm \\
0.06^{\mathrm{a}}\end{array}$ & $\begin{array}{l}1.31 \pm \\
0.26^{\mathrm{bc}}\end{array}$ & $\begin{array}{c}269.28 \pm \\
9.82^{c}\end{array}$ & $\begin{array}{c}64.66 \pm \\
2.06^{\mathrm{c}}\end{array}$ & $\begin{array}{c}25.90 \pm \\
0.22^{\mathrm{b}}\end{array}$ & $0.08^{\mathrm{a}}$ & $\begin{array}{c}0.27 \pm \\
0.07^{\text {a }}\end{array}$ & $\begin{array}{c}169.99 \pm \\
2.16^{\text {be }}\end{array}$ & $\begin{array}{l}.58 \\
18^{c}\end{array}$ & $\begin{array}{c}0.12 \pm \\
0.05^{\mathrm{a}}\end{array}$ & $\begin{array}{c}0.49 \pm \\
0.03^{\mathrm{a}}\end{array}$ & $\begin{array}{l}1470.98 \\
\pm 12.06^{c} \\
\end{array}$ \\
\hline C-06 & $\begin{array}{c}0.15 \pm \\
0.03^{\mathrm{a}}\end{array}$ & $\begin{array}{c}516.91 \pm \\
6.37^{\mathrm{d}}\end{array}$ & $=\begin{array}{c}12.45 \pm \\
0.61^{\mathrm{a}}\end{array}$ & $\begin{array}{l}3.56 \pm \\
0.13^{\text {bd }}\end{array}$ & $\begin{array}{l}1825.46 \\
\pm 24.54^{\mathrm{d}}\end{array}$ & $\begin{array}{c}143.49 \pm \\
3.28^{\mathrm{ak}}\end{array}$ & $\begin{array}{c}101.67 \\
\pm 1.63^{\mathrm{ag}}\end{array}$ & $\begin{array}{c}852.45 \pm \\
7.85^{c}\end{array}$ & $\begin{array}{c}0.23 \pm \\
0.03^{\mathrm{a}}\end{array}$ & $\begin{array}{c}9.33 \pm \\
0.53^{\mathrm{a}}\end{array}$ & $\begin{array}{c}689.07 \\
\pm 10.59^{\mathrm{d}}\end{array}$ & $\begin{array}{c}1.62 \pm \\
0.13^{\mathrm{a}}\end{array}$ & $\begin{array}{c}5.62 \pm \\
0.28^{\mathrm{a}}\end{array}$ & $\begin{array}{c}671.95 \pm \\
9.04^{\mathrm{d}}\end{array}$ \\
\hline FC-0 & $\begin{array}{c}0.15 \pm \\
0.03^{\mathrm{a}}\end{array}$ & $\begin{array}{c}2543.73 \\
\pm 68.17 \mathrm{e}\end{array}$ & $\begin{array}{c}17.92 \pm \\
0.29^{\mathrm{a}}\end{array}$ & $\begin{array}{l}2.71 \pm \\
0.26^{\text {be }}\end{array}$ & $\begin{array}{c}594.31 \pm \\
13.09^{\mathrm{e}}\end{array}$ & $\begin{array}{l}1220.81 \\
\pm 25.54^{\mathrm{d}}\end{array}$ & $\begin{array}{c}940.88 \\
\pm 14.94^{c}\end{array}$ & $\begin{array}{l}173 \\
\pm 9\end{array}$ & $\begin{array}{c}0.67 \pm \\
0.13^{\mathrm{a}}\end{array}$ & $\begin{array}{c}5.13 \pm \\
0.17^{\mathrm{a}}\end{array}$ & $\begin{array}{c}788.00 \\
\pm 50.88^{\mathrm{e}} \\
\end{array}$ & $\begin{array}{c}4.63 \pm \\
0.61^{\mathrm{a}}\end{array}$ & $\begin{array}{c}2.31 \pm \\
0.57^{\mathrm{a}}\end{array}$ & $\begin{array}{l}0.96 \\
.06^{\mathrm{a}}\end{array}$ \\
\hline $\mathrm{C}-$ & $\begin{array}{c}0.15 \pm \\
0.03^{\mathrm{a}}\end{array}$ & $\begin{array}{c}908.18 \pm \\
9.07^{\mathrm{fm}}\end{array}$ & $\begin{array}{c}27.61 \pm \\
1.35^{\mathrm{a}}\end{array}$ & $\begin{array}{c}5.57 \pm \\
0.80^{\mathrm{f}}\end{array}$ & & $\begin{array}{c}166.77 \pm \\
1.15^{\text {aj }}\end{array}$ & $\begin{array}{c}105.54 \\
\pm 2.29^{\mathrm{ag}} \\
\end{array}$ & $\begin{array}{c}749.54 \pm \\
0.69^{\mathrm{e}}\end{array}$ & $\begin{array}{l}12 \pm \\
04^{a} \\
\end{array}$ & $\begin{array}{l}3.22 \\
4.27^{c}\end{array}$ & $\begin{array}{l}68 \\
88^{\text {ef }} \\
\end{array}$ & $\begin{array}{c} \pm \\
3^{a}\end{array}$ & $\begin{array}{c}8.43 \pm \\
0.86^{\mathrm{a}}\end{array}$ & $\begin{array}{c}677.17 \pm \\
9.04^{\mathrm{de}}\end{array}$ \\
\hline $\mathrm{E}-13$ & $\begin{array}{c}0.13 \pm \\
0.06^{\mathrm{a}}\end{array}$ & \begin{tabular}{|l|}
1081.58 \\
$\pm 56.53^{\mathrm{g}}$ \\
\end{tabular} & $\begin{array}{c}6.62 \pm \\
0.22^{\mathrm{a}} \\
\end{array}$ & $\begin{array}{c}0.46 \pm \\
0.02^{\mathrm{g}} \\
\end{array}$ & $\begin{array}{l}409.72 \\
36.00^{\mathrm{g}} \\
\end{array}$ & $\begin{array}{c}448.58 \pm \\
6.82^{\mathrm{e}}\end{array}$ & $\begin{array}{c}330.74 \\
\pm 27.86^{6}\end{array}$ & $\begin{array}{c}115.46 \pm \\
4.83^{\mathrm{bf}}\end{array}$ & $\begin{array}{l}0.12 \pm \\
0.04^{a}\end{array}$ & $\begin{array}{c}100.47 \pm \\
1.82^{\mathrm{df}}\end{array}$ & $\begin{array}{c}648.82 \\
\pm 5.18^{\mathrm{dg}} \\
\end{array}$ & $\begin{array}{c}1.30 \pm \\
0.20^{\mathrm{a}} \\
\end{array}$ & $\begin{array}{c}17.36 \pm \\
1.72^{\mathrm{a}}\end{array}$ & $\begin{array}{r}388.78 \pm \\
14.07^{\mathrm{f}}\end{array}$ \\
\hline$C_{-}$ & $\begin{array}{c}0.15 \pm \\
0.03^{\mathrm{a}}\end{array}$ & $\begin{array}{c}889.41 \pm \\
7.58^{\mathrm{hm}}\end{array}$ & $\begin{array}{c}11.66 \pm \\
0.71^{\mathrm{a}}\end{array}$ & $\begin{array}{c}7.35 \pm \\
0.66^{\mathrm{h}}\end{array}$ & $\begin{array}{l}339.45 \\
16.36^{\mathrm{h}}\end{array}$ & $\begin{array}{c}214.99 \pm \\
4.38^{\mathrm{jf}}\end{array}$ & $\begin{array}{l}154.20 \\
\pm 1.15^{\mathrm{a}}\end{array}$ & $\begin{array}{c}295.24 \pm \\
5.09^{g}\end{array}$ & $\begin{array}{c}0.27 \pm \\
0.07^{\mathrm{a}}\end{array}$ & $\begin{array}{c}14.41 \pm \\
0.89^{\mathrm{a}}\end{array}$ & $\begin{array}{c}1008.53 \\
\pm 55.19^{\mathrm{h}}\end{array}$ & $\begin{array}{c}1.50 \pm \\
0.13^{\mathrm{a}}\end{array}$ & $\begin{array}{c}5.29 \pm \\
0.57^{\mathrm{a}}\end{array}$ & $\begin{array}{r}843.71 \pm \\
25.12^{\mathrm{g}}\end{array}$ \\
\hline EC-20 & $\begin{array}{c}0.15 \pm \\
0.03^{\mathrm{a}}\end{array}$ & $\begin{array}{c}308.84 \pm \\
1.95^{i}\end{array}$ & $\begin{array}{c}10.34 \pm \\
0.35^{a}\end{array}$ & $\begin{array}{c}1.00 \pm \\
0.13^{\mathrm{i}} \\
\end{array}$ & $\begin{array}{c}949.57 \pm \\
73.64^{\mathrm{i}}\end{array}$ & $\begin{array}{c}685.22 \pm \\
26.60^{\mathrm{g}}\end{array}$ & $\begin{array}{c}480.60 \\
\pm 19.66^{\circ}\end{array}$ & $\begin{array}{c}1094.20 \\
\pm 1.55^{\mathrm{h}} \\
\end{array}$ & $\begin{array}{l}0.27 \pm \\
0.07^{\text {a }}\end{array}$ & $\begin{array}{c}4.77 \pm \\
0.21^{\mathrm{a}} \\
\end{array}$ & $\begin{array}{r}6.97 \\
8.42^{\mathrm{i}} \\
\end{array}$ & $\begin{array}{c}5.42 \pm \\
0.34^{\mathrm{a}} \\
\end{array}$ & $\begin{array}{c}3.80 \pm \\
0.57^{\mathrm{a}} \\
\end{array}$ & $\begin{array}{l}1676.97 \\
\pm 70.35^{\mathrm{h}} \\
\end{array}$ \\
\hline $\mathrm{E}-27$ & $\begin{array}{c}0.06 \pm \\
0.03^{\mathrm{a}}\end{array}$ & $\begin{array}{c}308.84 \pm \\
1.95^{\mathrm{a}}\end{array}$ & $\begin{array}{c}6.57 \pm \\
0.42^{\mathrm{a}}\end{array}$ & $\begin{array}{c}1.78 \pm \\
0.26^{j}\end{array}$ & $\begin{array}{c}491.32 \pm \\
31.09^{j}\end{array}$ & $\begin{array}{c}50.85 \pm \\
1.78^{\mathrm{ch}} \\
\end{array}$ & $\begin{array}{r}35.73 \pm \\
2.01^{\mathrm{bf}}\end{array}$ & $\begin{array}{c}149.79 \pm \\
2.41^{\mathrm{bi}}\end{array}$ & $\begin{array}{c}0.12 \pm \\
0.04^{\text {a }}\end{array}$ & $\begin{array}{c}112.31 \pm \\
2.04^{\mathrm{ef}}\end{array}$ & $\begin{array}{l}271.89 \\
\pm 3.00^{\mathrm{a}} \\
\end{array}$ & $\begin{array}{c}1.07 \pm \\
0.20^{\mathrm{a}}\end{array}$ & $\begin{array}{c}8.10 \pm \\
1.14^{\mathrm{a}}\end{array}$ & $\begin{array}{r}813.53 \pm \\
55.28^{\mathrm{gi}}\end{array}$ \\
\hline $\mathrm{E}-31$ & $\begin{array}{c}0.06 \pm \\
0.03^{\mathrm{a}} \\
\end{array}$ & $\begin{array}{c}365.10 \pm \\
1.14^{\mathrm{an}}\end{array}$ & $\begin{array}{c}13.27 \pm \\
0.39^{\mathrm{a}}\end{array}$ & $\begin{array}{c}0.85 \pm \\
0.26^{\mathrm{k}} \\
\end{array}$ & $\begin{array}{c}759.66 \pm \\
24.55^{\mathrm{k}} \\
\end{array}$ & $\begin{array}{c}96.33 \pm \\
3.45^{\mathrm{cl}} \\
\end{array}$ & $\begin{array}{c}47.57 \pm \\
1.50^{\mathrm{bg}} \\
\end{array}$ & $\begin{array}{c}878.06 \pm \\
19.06^{\mathrm{j}} \\
\end{array}$ & $\begin{array}{l}0.12 \pm \\
0.04^{a}\end{array}$ & $\begin{array}{c}21.02 \pm \\
0.16^{\mathrm{a}}\end{array}$ & \begin{tabular}{|l|}
243.76 \\
$\pm 3.87^{\mathrm{a}}$ \\
\end{tabular} & $\begin{array}{c}1.70 \pm \\
0.06^{\mathrm{a}} \\
\end{array}$ & $\begin{array}{c}4.13 \pm \\
0.28^{\mathrm{a}}\end{array}$ & $\begin{array}{c}901.15 \pm \\
8.04^{\mathrm{j}}\end{array}$ \\
\hline SEC- & $\begin{array}{c}0.15 \pm \\
0.03^{\mathrm{a}}\end{array}$ & $\begin{array}{c}407.53 \pm \\
2.58^{\text {jn }}\end{array}$ & $\begin{array}{c}3.79 \pm \\
0.64^{\mathrm{a}}\end{array}$ & $\begin{array}{c}3.48 \pm \\
0.80^{1}\end{array}$ & $\begin{array}{c}592.42 \pm \\
19.63^{\text {el }}\end{array}$ & $\begin{array}{c}192.14 \pm \\
2.21^{\mathrm{ijk}}\end{array}$ & $\begin{array}{l}126.24 \\
\pm 1.45^{\mathrm{a}}\end{array}$ & $\begin{array}{c}97.38 \pm \\
4.31^{\mathrm{bk}}\end{array}$ & $\begin{array}{c}0.12 \pm \\
0.04^{\mathrm{a}}\end{array}$ & $\begin{array}{c}23.06 \pm \\
0.19^{\mathrm{a}}\end{array}$ & $\begin{array}{l}332.24 \\
\pm 4.11^{\mathrm{j}}\end{array}$ & $\begin{array}{l}1.90 \pm \\
0.20^{\mathrm{a}}\end{array}$ & $\begin{array}{c}3.30 \pm \\
0.57^{\mathrm{a}}\end{array}$ & $\begin{array}{c}487.42 \pm \\
9.04^{\mathrm{bk}}\end{array}$ \\
\hline $\begin{array}{l}\text { ax } \\
\text { alue }\end{array}$ & $\begin{array}{c}0.17 \pm \\
0.07\end{array}$ & $\begin{array}{r}2543.73 \\
\pm 68.17 \\
\end{array}$ & $\begin{array}{c}27.61 \pm \\
1.35\end{array}$ & $\begin{array}{c}96.97 \pm \\
1.60\end{array}$ & \begin{tabular}{|l|}
4080.82 \\
\pm 130.92 \\
\end{tabular} & $\begin{array}{c}1220.81 \\
\pm 25.54 \\
\end{array}$ & $\begin{array}{r}940.88 \\
\pm 14.94 \\
\end{array}$ & $\begin{array}{c}1736.59 \\
\pm 94.90\end{array}$ & $\begin{array}{c}0.67 \pm \\
0.13\end{array}$ & $\begin{array}{r}1483.22 \\
\pm 14.27\end{array}$ & $\begin{array}{r}1008.53 \\
\pm 55.19\end{array}$ & $\begin{array}{c}5.42 \pm \\
0.34\end{array}$ & $\begin{array}{c}17.36 \pm \\
1.72\end{array}$ & $\begin{array}{l}1676.97 \\
\pm 70.35\end{array}$ \\
\hline $\begin{array}{l}\text { Min } \\
\text { value }\end{array}$ & $\begin{array}{c}0.06 \pm \\
0.03\end{array}$ & $\begin{array}{c}5.44 \pm \\
0.63\end{array}$ & $\begin{array}{c}0.43 \pm \\
0.06\end{array}$ & $\begin{array}{l}0.46 \\
0.02\end{array}$ & $\begin{array}{c}269.28 \pm \\
9.82\end{array}$ & $\begin{array}{c}50.85 \\
1.78\end{array}$ & $\begin{array}{c}25.90 \pm \\
0.22\end{array}$ & $\begin{array}{c}0.94 \pm \\
0.08\end{array}$ & $\begin{array}{c}0.12 \pm \\
0.04\end{array}$ & $\begin{array}{c}4.77 \pm \\
0.21\end{array}$ & $\begin{array}{r}225.07 \\
\pm 1.62\end{array}$ & $\begin{array}{c}0.12 \pm \\
0.05\end{array}$ & $\begin{array}{c}0.49 \pm \\
0.03\end{array}$ & $\begin{array}{c}388.78 \pm \\
14.07\end{array}$ \\
\hline
\end{tabular}

Table 3: Phenolic acids composition of twelve different accessions from India ( $\mu \mathrm{g} / \mathrm{g}$ of Dried Material).

*Data are represented as the mean \pm Standard deviation $(n=3)$. Values in the same column that are followed by different superscript letters are significantly different $(\mathrm{p}<0.05)$.

*Abbreviations: ChlA: Chlorogenic Acid, FerA: Ferulic Acid, CafA: Caffeic Acid, GalA: Gallic Acid, VanA: Vanillic Acid, P-CA: $p$-Coumaric Acid, o-CA: o-Coumaric Acid, ProtA: Protocatechuic Acid, GenA: Gentisic Acid, SalA: Salicylic Acid, p-HBA: p-hydrozybenzoic Acid, 2,4 DHBA: 2,4- Dihydroxy Benzoic Acid, t-CinA: t-cinnamic Acid, SyrA: Syringic Acid. 


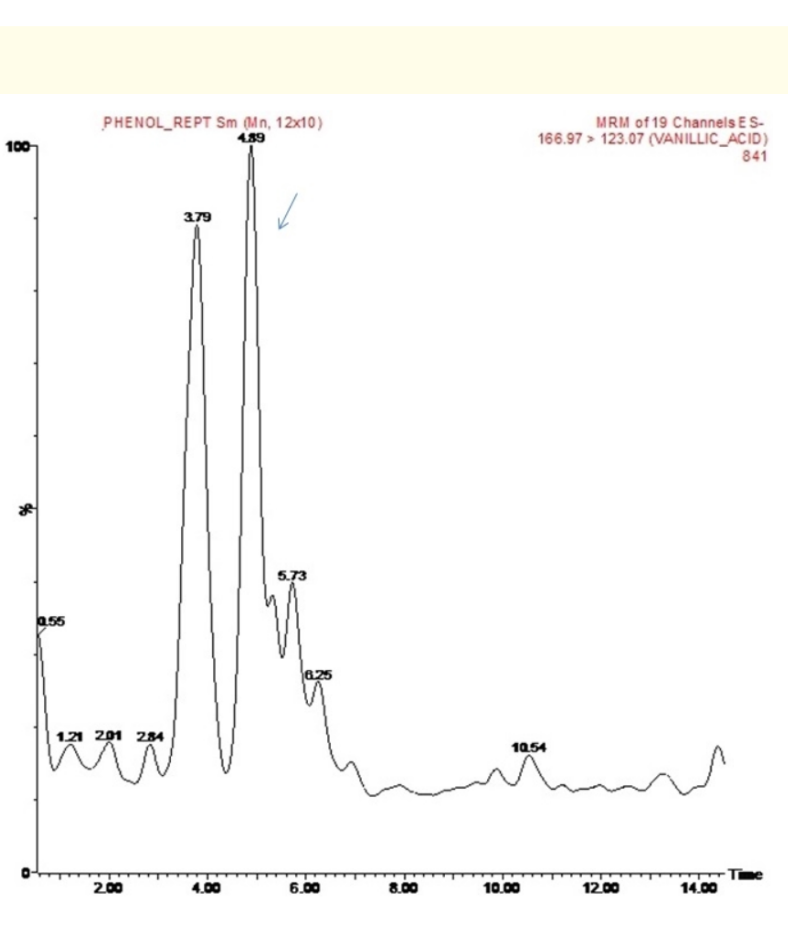

Figure 2: Chromatograms obtained using MRM mode (multiple reaction monitoring) for vanillic acid from S. edule extracts.
Catechin was present in major quantity compared to other flavonoids among accessions. Accessions SEC-05 had high amounts of quercetin $(7.11 \pm 1.44 \mu \mathrm{g} / \mathrm{g})$ and catechin $(16.01 \pm 1.46 \mu \mathrm{g} / \mathrm{g})$, accession SEC-09 had high amounts of quercetin $(11.70 \pm 0.72 \mu \mathrm{g} / \mathrm{g})$, hespertin $(8.72 \pm 0.86 \mu \mathrm{g} / \mathrm{g})$ and catechin $(21.06 \pm 1.45 \mu \mathrm{g} / \mathrm{g})$ comparatively, accession SEC-11 also had high amounts of catechin $(28.65 \pm 1.46 \mu \mathrm{g} / \mathrm{g})$ and quercetin $(9.62 \pm 1.44 \mu \mathrm{g} / \mathrm{g})$. Accession SEC-36 had the highest amount of catechin present $(75.83 \pm 4.37$ $\mu \mathrm{g} / \mathrm{g}$ ) (Table 5). The chromatogram of catechin for the extract of S. edule accession SEC-11 is represented with the standard curve obtained using multiple reaction monitoring is provided in Figure 3.

Flavonoid content is usually dependent on the cultivars and growing conditions as they are produced in direct response to environmental conditions. Therefore, the differences in concentration in the collected accessions was observed [20,21]. Catechins, a disease fighting flavonoid, which are a group of flavanols are found in various fruits and vegetables derived from plants. It is one of the potent antioxidant compound present. The extract of o. linearis was found to have high amounts of catechin compared to other flavanols $(0.113 \pm 0.03 \mathrm{mg} / \mathrm{gm})$ [22]. About four flavonoids were detected in a study conducted by Siciliano., et al. (2004) in

\begin{tabular}{|l|c|c|c|c|c|c|}
\hline \multicolumn{1}{|c|}{ Compound } & Formula/ass & Parent m/z & Cone Voltage & Daughters & Collision Energy & Ion Mode \\
\hline Apigenin & 270 & 268.97 & 46 & 107.04 & 30 & ES- \\
\hline Catechin & 290 & 289.03 & 38 & 245.15 & 12 & ES- \\
\hline Hesperetin & 302 & 300.97 & 42 & 286.15 & 16 & ES- \\
\hline Leutoline & 286 & 284.97 & 54 & 150.99 & 26 & ES- \\
\hline Myrcetin & 318 & 317.03 & 42 & 151.06 & 28 & ES- \\
\hline Naringenin & 272 & 271.03 & 34 & 151.00 & 16 & ES- \\
\hline Quercetin & 302 & 301.03 & 36 & 151.12 & 20 & ES- \\
\hline Rutin & 610 & 609.10 & 60 & 300.20 & 42 & ES- \\
\hline Umbelliferone & 162.14 & 161.04 & 42 & 133.07 & 18 & ES- \\
\hline
\end{tabular}

Table 4: Flavanoids MRM details.

S. edule fruits collected from Monasterace (RC), Italy and those were, vicenin- 2 , apigenin 6-C- $\hat{a}$-D-glucopyranosyl-8-C- $\hat{a}$-D-apiofuranoside, vitexin and luteolin 7-0-rutinoside in trace amounts using HPLC-PDA-ESI-MS detection method. Substantially, LC-MS method developed in our study is more sensitive than the other reported methods as we could detect nine flavonoids in minimal amounts. Though S. edule was found to have low concentrations of flavonoids comparatively to other plant species, the distribution profile of each flavonoid among all accessions decreased in the order SEC-36 > SEC-11 > SEC-09 > SEC-20 > SEC-06 > SEC-05 > SEC- 


\begin{tabular}{|c|c|c|c|c|c|c|c|c|c|}
\hline Acc No. & Quercetin & Hespertin & Catechin & Luteolene & Naringenin & Apigenin & Umbeliferon & Rutin & Myricetin \\
\hline SEC-01 & $2.92 \pm 0.72^{a}$ & $4.73 \pm 0.43^{\mathrm{aj}}$ & $2.53 \pm 0.04^{\mathrm{a}}$ & $0.27 \pm 0.03^{\mathrm{a}}$ & $0.62 \pm 0.11^{\mathrm{a}}$ & $0.31 \pm 0.07^{\mathrm{a}}$ & $0.14 \pm 0.05^{a}$ & $3.46 \pm 0.16^{a}$ & $1.60 \pm 0.27^{\mathrm{a}}$ \\
\hline SEC-03 & $1.25 \pm 0.02^{\mathrm{a}}$ & $0.75 \pm 0.04^{\mathrm{b}}$ & $5.90 \pm 1.46^{\mathrm{b}}$ & $1.1 \pm 1.19^{\mathrm{abd}}$ & $0.18 \pm 0.03^{\mathrm{a}}$ & $0.31 \pm 0.07^{\mathrm{a}}$ & $0.14 \pm 0.05^{\mathrm{a}}$ & $3.83 \pm 0.32^{\mathrm{a}}$ & $2.08 \pm 0.27^{\mathrm{a}}$ \\
\hline SEC-06 & $\begin{array}{l}5.43 \pm \\
0.72^{\mathrm{bc}}\end{array}$ & $0.75 \pm 0.04^{\mathrm{bd}}$ & $13.48 \pm 1.46^{\mathrm{d}}$ & $2.93 \pm 0.31^{\text {bcde }}$ & $0.18 \pm 0.03^{\mathrm{a}}$ & $0.88 \pm 0.15^{a}$ & $1.02 \pm 0.08^{a}$ & $1.49 \pm 0.16^{\mathrm{bc}}$ & $2.08 \pm 0.27^{\mathrm{a}}$ \\
\hline SEC-09 & $\begin{array}{c}11.70 \pm \\
0.72^{\mathrm{d}}\end{array}$ & $8.72 \pm 0.86^{e}$ & $21.06 \pm 1.45^{\mathrm{e}}$ & $0.92 \pm 0.16^{\mathrm{a}}$ & $0.37 \pm 0.01^{\mathrm{a}}$ & $0.39 \pm 0.03^{\mathrm{a}}$ & $0.14 \pm 0.05^{\mathrm{a}}$ & $\begin{array}{l}0.56 \pm \\
0.03^{\text {bd }}\end{array}$ & $0.48 \pm 0.01^{\mathrm{a}}$ \\
\hline SEC-11 & $9.62 \pm 1.44^{\mathrm{e}}$ & $3.98 \pm 0.43^{\mathrm{a}}$ & $28.65 \pm 1.46^{f}$ & $1.19 \pm 0.15^{\text {ade }}$ & $1.18 \pm 0.10^{\mathrm{a}}$ & $0.39 \pm 0.03^{\mathrm{a}}$ & $0.46 \pm 0.08^{\mathrm{a}}$ & $1.21 \pm 0.16^{\mathrm{be}}$ & $2.08 \pm 0.27^{\mathrm{a}}$ \\
\hline SEC-13 & $1.25 \pm 0.02^{\mathrm{a}}$ & $3.98 \pm 0.43^{\mathrm{a}}$ & $8.42 \pm 1.46^{\mathrm{g}}$ & $1.83 \pm 0.31^{\text {ade }}$ & $0.37 \pm 0.01^{\mathrm{a}}$ & $0.31 \pm 0.07^{\mathrm{a}}$ & $0.28 \pm 0.05^{a}$ & $1.49 \pm 0.16^{\mathrm{bf}}$ & $2.08 \pm 0.27^{\mathrm{a}}$ \\
\hline SEC-18 & $1.25 \pm 0.02^{\mathrm{a}}$ & $0.75 \pm 0.04^{\mathrm{bf}}$ & $5.05 \pm 0.03^{\mathrm{bh}}$ & $0.92 \pm 0.16^{\mathrm{a}}$ & $0.43 \pm 0.10^{\mathrm{a}}$ & $0.13 \pm 0.01^{\mathrm{a}}$ & $0.88 \pm 0.08^{a}$ & $\begin{array}{c}2.43 \pm \\
0.32^{\text {acdefj }}\end{array}$ & $1.60 \pm 0.27^{\mathrm{a}}$ \\
\hline SEC-27 & $2.51 \pm 0.05^{\mathrm{a}}$ & $0.75 \pm 0.04^{\mathrm{bh}}$ & $10.95 \pm 1.45^{j}$ & $1.19 \pm 0.15^{\mathrm{aeg}}$ & $0.99 \pm 0.10^{\mathrm{a}}$ & $0.26 \pm 0.02^{\mathrm{a}}$ & $0.28 \pm 0.05^{a}$ & $\begin{array}{c}0.56 \pm \\
0.03^{\text {bjkh }}\end{array}$ & $0.48 \pm 0.01^{\mathrm{a}}$ \\
\hline SEC-31 & $\begin{array}{l}7.94 \pm \\
0.72^{\text {efg }} \\
\end{array}$ & $0.75 \pm 0.04^{\mathrm{bi}}$ & $8.43 \pm 1.45^{\mathrm{gk}}$ & $2.93 \pm 0.31^{\mathrm{dfg}}$ & $1.36 \pm 0.10^{\mathrm{a}}$ & $0.13 \pm 0.01^{\mathrm{a}}$ & $0.28 \pm 0.05^{\mathrm{a}}$ & $\begin{array}{l}0.84 \pm \\
0.05^{\mathrm{bijk}} \\
\end{array}$ & $2.08 \pm 0.27^{\mathrm{a}}$ \\
\hline SEC-36 & $2.92 \pm 0.72^{\mathrm{a}}$ & $6.48 \pm 0.86^{j}$ & $75.83 \pm 4.37^{1}$ & $0.27 \pm 0.03^{\mathrm{a}}$ & $0.37 \pm 0.01^{\mathrm{a}}$ & $0.97 \pm 0.07^{\mathrm{a}}$ & $0.60 \pm 0.08^{\mathrm{a}}$ & $\begin{array}{c}2.34 \pm \\
0.16^{\text {acdefk }}\end{array}$ & $0.96 \pm 0.03^{\mathrm{a}}$ \\
\hline $\begin{array}{l}\text { Max } \\
\text { value }\end{array}$ & $\begin{array}{c}11.70 \pm \\
0.72\end{array}$ & $8.72 \pm 0.86$ & $75.83 \pm 4.37$ & $4.49 \pm 0.31$ & $1.18 \pm 0.10$ & $\begin{array}{c}0.9797 \pm \\
0.07\end{array}$ & $1.02 \pm 0.08$ & $19.43 \pm 0.64$ & $2.08 \pm 0.27$ \\
\hline $\begin{array}{l}\text { Min } \\
\text { value }\end{array}$ & $1.25 \pm 0.02$ & $0.75 \pm 0.04$ & $2.53 \pm 0.04$ & $0.27 \pm 0.03$ & $0.18 \pm 0.03$ & $0.13 \pm 0.01$ & $0.14 \pm 0.05$ & $0.28 \pm 0.01$ & $0.48 \pm 0.01$ \\
\hline
\end{tabular}

Table 5: Flavonoids composition of twelve different accessions from India ( $\mu \mathrm{g} / \mathrm{g}$ of Dried Material).

Data are represented as the mean \pm Standard deviation $(n=3)$. Values in the same column that are followed by different superscript letters are significantly different $(\mathrm{p}<0.05)$.

\section{$31>$ SEC-13 > SEC-27 > SEC-01 > SEC-03 > SEC-18}

Principle component analysis of phenolic acids and flavonoids

The LC-MS data is confirmed by Principal component analysis, which showed that the accessions SEC-09, SEC-11 and SEC-20 forms a distinct individual unclustered accessions compared to other remaining accessions showing the presence of high amount of phenolic acids and flavonoids. The components PC1 and PC2 showed about $30 \%$ and $18 \%$ variation. The total variation of $90 \%$ was observed by the first six principal components (i.e., 30, 18, 13, 11,11 and 7\%). PCA is considered to be a powerful tool for identification of data patterns and useful in analysis of data highlighting the similarities and differences in a group, and provides the plots for distribution of samples and variables employed on the principal components respectively.

Ferulic acid, protocatechuic acid, $p$-courmaric acid, gentic acid, benzoic acid, caffeic acid, $p$-hydroxybenzoic acid, vanillic acid, salicylic acid and myrecetin had higher coefficients on the axis of the first PC as compared to the other axis. Such an analysis provides information about the correlations as well as dependencies of metabolites among accessions [23]. The scatter plots of score and loadings of PC1 and PC2 is shown in the Figure 4A and Figure 4B depicting the formation of one major cluster towards left. Similar studies have been conducted using wheat varieties and vegetable oils $[24,25]$. Therefore, Exploration of PCA for the data obtained using LC-MS demonstrated that the method is useful for discrimina- 


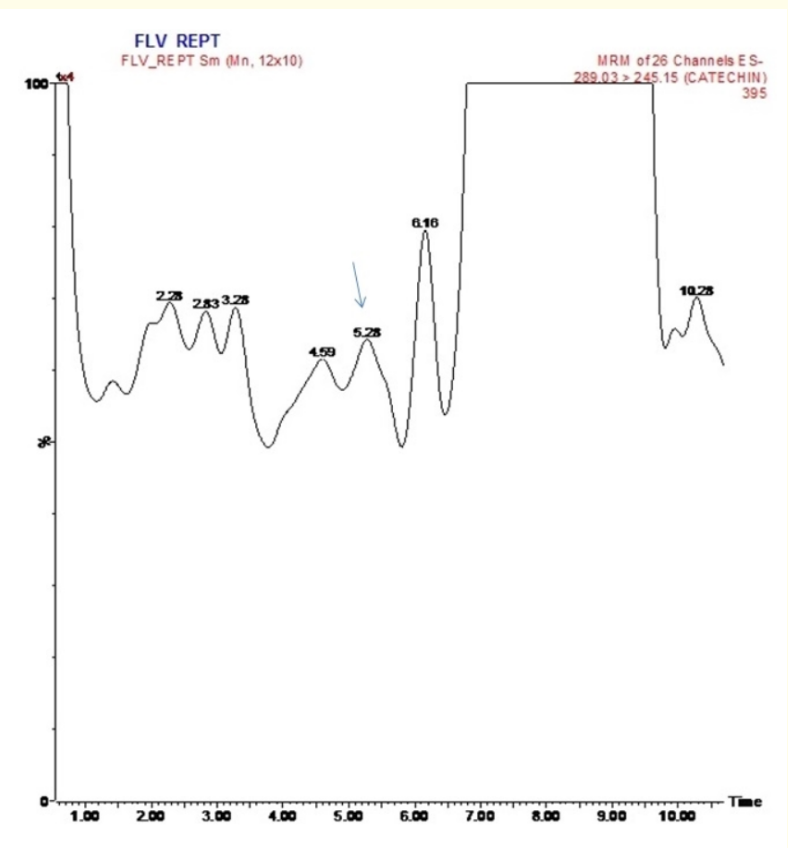

Figure 3: Chromatograms obtained using MRM mode (multiple reaction monitoring) for catechin from S. edule extracts.

tion of $S$. edule accessions. Quantification of phenolic acids and flavonoids in S. edule is important for application purpose as it affects the quality of fruits and simultaneously the antioxidant activities may be beneficial for improving health and preventing diseases.

\section{Conclusion}

We showed the first comprehensive study of variability of phenolic acids and flavonoids in the accessions collected from India. In total, fourteen phenolic acids and nine flavonoids were quantified by LC-MS in the methanolic extracts of $S$. edule, and the establishment of such a method showed well separation of compounds and are reproducible. With the aid of PCA, the LC-MS data were mined for similarity and differences in phytochemical composition between accessions. Despite the numerous methods for polyphenols detection, the validated and optimized method in $S$. edule is still lacking. Therefore, this method is suitable for determination of phenolic acids and flavonoids for high efficiency and can help in genetic grouping of landraces as well as for developing more efficient strategies to gain a greater knowledge for future breeding programs.
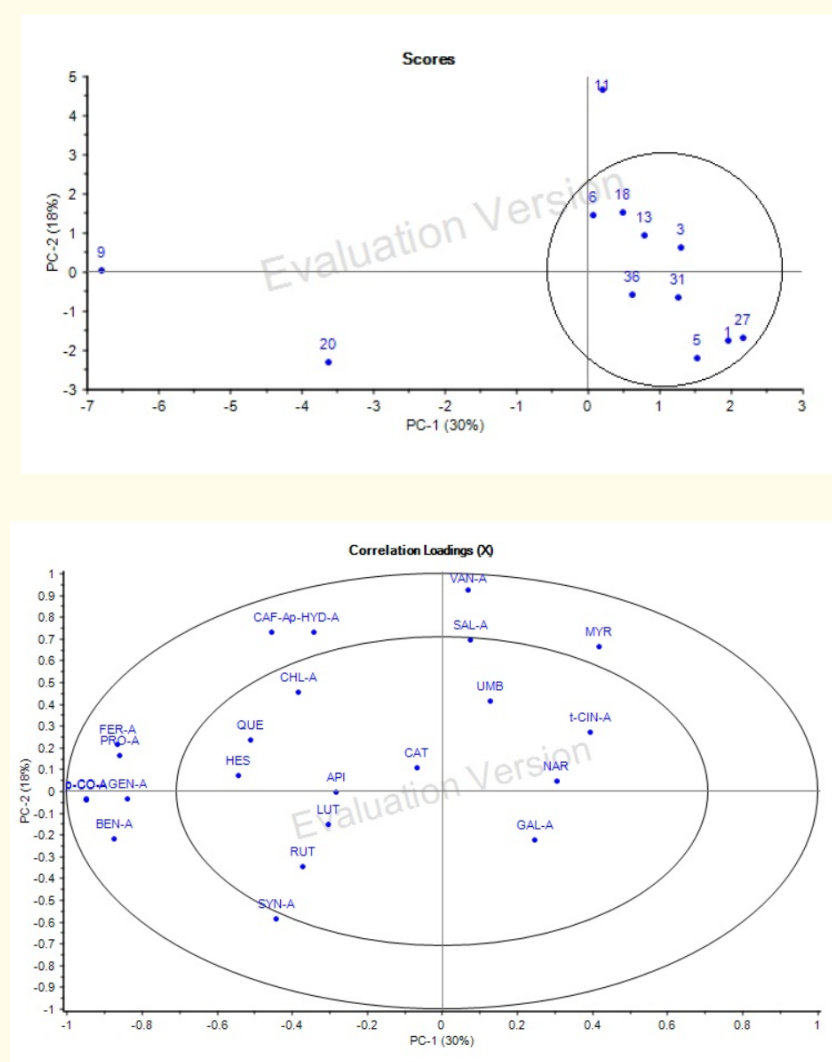

Figure 4: Principal component analysis (PCA) results for phenolic acids and flavonoids present in $S$. edule accessions (Score (A) and loadings (B) plots of PC1 and PC2).

\section{Acknowledgement}

No funding was received for conducting this study. Authors thank the management of JAIN (Deemed_To_Be_University), School of Sciences, Department of Biotechnology and ICAR-Indian Institute of Horticultural Research, Division of Plant Physiology and Biochemistry for providing the necessary facilities to conduct the present study.

\section{Conflict of Interest}

The authors have no conflicts of interest to declare that are relevant to the content of this article.

\section{Bibliography}

1. Huang Z., et al. "Phenolic compound profile of selected vegetables frequently consumed by African Americans in the southeast United States". Food Chemistry 103 (2007): 1395-1402. 
2. Ghasemzadeh A and Ghasemzadeh N. "Flavonoids and phenolic acids: Role and biochemical activity in plants and human". Journal of Medicinal Plant Research 5.31 (2011): 6697-6703.

3. Tsao R and Deng Z. "Separation procedures for naturally occurring antioxidant photochemicals". Journal of Chromatography B 812 (2004): 85-99.

4. Tapas AR., et al. "Flavonoids as Nutraceuticals: A Review". Tropical Journal of Pharmaceutical Research 7.3 (2008): 10891099.

5. Shahidi F and Wanasundara PK. "Phenolic antioxidants". Critical Reviews in Food Science and Nutrition 32 (1992): 67-103.

6. Tim TP and Lamb AJ. "Antimicrobial activity of flavonoids". International Journal of Antimicrobial Agents 26 (2005): 343356.

7. Wei H., et al. "Inhibitory effect of epigenin, a plant flavonoid, on epidermal ornithine decarboxylase and skin tumor promotion in mice". Cancer Research 50 (1990): 499-502.

8. Migliaccio S and Anderson JB. "Isoflavones and skeletal health: Are these molecules ready for clinical application". Osteoporosis International 14 (2003): 361-368.

9. Jain JR., et al. "Standardization of DNA isolation and RAPDPCR protocol from Sechium edule". International Journal of Advancement in Life Sciences Research 8.3 (2015): 359-363.

10. Jain JR., et al. "A comparative assessment of morphological and molecular diversity among Sechium edule (Jacq.) Sw. accessions in India". 3 Biotech 7 (2017): 106.

11. Shivashankar S., et al. "Role of phenolic acids and enzymes of phenylpropanoid pathway in resistance of chayote fruit (Sechium edule) against infestation by melon fly, Bactrocera cucurbitae". Annals of Applied Biology 72 (2015): 1-14.

12. Siciliano T., et al. "Study of flavonoids of Sechium edule (Jacq) Swartz (Cucurbitaceae) different edible organs by liquid chromatography photodiode array mass spectrometry". Journal of Agricultural and Food Chemistry 52.21 (2004): 6510-6515.

13. Weidner S., et al. "Changes in endogenous phenolic acids during development of Secale cereale caryopses and after dehydration treatment of unripe rye grains". Plant Physiology and Biochemistry 38 (2000): 595-602.
14. Chen H., et al. "Separation and determination of flavonoids and other phenolic compounds in cranberry juice by highperformance liquid chromatography". Journal of Chromatography 913 (2001): 387-395.

15. Middha SK., et al. "In silico exploration of cyclooxygenase inhibitory activity of natural compounds found in Myrica nagi using LC-MS". Symbiosis (2016).

16. Vinayagam R. "Preventive effect of Syringic acid on hepatic marker enzymes and lipid profile against acetaminopheninduced hepatotoxicity rats". International Journal of Pharmaceutical and Biological Archive 1 (2010): 393-398.

17. Mussatto G., et al. "Ferulic and p-coumaric acids extraction by alkaline hydrolysis of brewer's spent grain". Industrial Crops and Products 25 (2007): 231-237.

18. Ramadoss K., et al. "Isolation, characterization, and RP-HPLC estimation of P-coumaric acid from methanolic extract of Durva Grass (Cynodondactylon Linn.) (Pers.)". International Journal of Analytical Chemistry (2007): 1-7.

19. Stommel JR., et al. "Phenolic Acid Content and Composition of Eggplant Fruit in a Germplasm Core Subset". Journal of the American Society for Horticultural Science 128.5 (2003): 704710.

20. Caldwell CR., et al. "Effect of temperature, elevated carbon dioxide, and drought during seed development on the isoflavone content of dwarf soybean [Glycine max (L.) Merrill] grown in controlled environments". Journal of Agricultural and Food Chemistry 53 (2005): 1125-1129.

21. Harnly JM., et al. "Flavonoid Content of U.S. Fruits, Vegetables and Nuts". Journal of Agricultural and Food Chemistry 54 (2006): 9966-9977.

22. Seal T. "Quantitative HPLC analysis of phenolic acids, flavonoids and ascorbic acid in four different solvent extracts of two wild edible leaves, Sonchus arvensis and Oenanthe linearis of North-Eastern region in India". Journal of Applied Pharmaceutical Science 6.2 (2016): 157-166.

23. Berrueta LA., et al. "Supervised pattern recognition in food analysis". Journal of Chromatography A 1158 (2007): 196-214. 
24. Levandia T., et al. "Principal component analysis of HPLC-MS/ MS patterns of wheat (Triticum aestivum) varieties". Proceedings of the Estonian Academy of Sciences 63.1 (2014): 86-92.

25. Farres-Cebrian M., et al. "HPLC-UV polyphenolic profiles in the classification of olive oils and other vegetable oils via principal component analysis". Separations 3.33 (2016): 1-13.

\section{Assets from publication with us}

- Prompt Acknowledgement after receiving the article

- Thorough Double blinded peer review

- Rapid Publication

- Issue of Publication Certificate

- High visibility of your Published work

Website: www.actascientific.com/

Submit Article: www.actascientific.com/submission.php

Email us: editor@actascientific.com

Contact us: +919182824667 